\title{
Efeito residual da adubação com composto orgânico sobre o crescimento e produção de alface ${ }^{(1)}$
}

\author{
Ricardo Henrique Silva Santos ${ }^{(2)}$, Franceli da Silva ${ }^{(2)}$, Vicente Wagner Dias Casali(2) e Alcides Reis Conde ${ }^{(3)}$
}

\begin{abstract}
Resumo - Este trabalho teve como objetivo avaliar o efeito residual do composto orgânico sobre o crescimento e a produção da alface. Plantas da cultivar Babá foram adubadas com cinco doses de composto orgânico $(0,0,22,8,45,6,68,4$ e 91,2 t/ha de matéria seca), na presença e na ausência de adubo mineral (643 kg/ha de 4-14-8 e duas aplicações de $30 \mathrm{~kg} / \mathrm{ha}$ de $\mathrm{N}$ em cobertura). O efeito residual foi avaliado em um segundo cultivo nas mesmas parcelas, sem adição de adubos. Doses crescentes de composto orgânico aumentaram a produção da cultura até $27.367 \mathrm{~kg} /$ ha de matéria fresca, obtida na dose mais elevada em plantas cultivadas entre 80 e 110 dias após a aplicação do composto. A aplicação de adubo mineral não apresentou efeito residual. Os efeitos residuais do composto orgânico expressaramse também pelo aumento linear dos teores de bases trocáveis, de $\mathrm{P}$ e da capacidade efetiva de troca de cátions, (CTC) do solo, que atingiram valores de $10,77 \mathrm{cmol}_{\mathrm{c}} / \mathrm{dm}^{3}, 461 \mathrm{mg} / \mathrm{dm}^{3}$ e $11,08 \mathrm{cmol}_{\mathrm{c}} / \mathrm{dm}^{3}$, respectivamente.
\end{abstract}

Termos para indexação: Lactuca sativa, adubação orgânica, adubação mineral, mineralização, efeitos residuais.

\section{Residual effect of organic compost on lettuce growth and yield}

Abstract - This paper aimed to evaluate the residual effect of organic compost fertilization on lettuce growth and yield. Seedlings of 'Babá' lettuce were fertilized with five doses of organic compost $(0.0$, $22.8,45.6,68.4$ and 91.2 ton/ha of dry matter), in the presence and in the absence of mineral fertilizer (643 kg/ha of 4-14-8 plus two side dressings of $30 \mathrm{~kg} / \mathrm{ha}$ of $\mathrm{N}$ ). The residual effect was evaluated by carrying out a second cultivation on the same plots with no fertilizer addition after the first cultivation. Fertilization with organic compost increased the second cultivation yield up to $27,367 \mathrm{~kg} / \mathrm{ha}$ of fresh weight at the highest dose, in plants grown between 80 and 110 days after organic compost application. The mineral fertilization showed no residual effect. The residual effect also resulted in increases in soil exchangeable bases, $\mathrm{P}$, and effective soil cation exchange capacity (CEC) reaching values of $10.77 \mathrm{cmol}_{\mathrm{c}} / \mathrm{dm}^{3}, 461 \mathrm{mg} / \mathrm{dm}^{3}$ and $11.08 \mathrm{cmol}_{\mathrm{c}} / \mathrm{dm}^{3}$, respectively.

Index terms: Lactuca sativa, organic fertilizers, inorganic amendments, mineralization, residual effects.

\section{Introdução}

O mercado de produtos orgânicos vem crescendo no Brasil e no mundo a uma taxa de até $50 \%$ ao ano. Neste contexto, o cultivo de hortaliças com adu-

(1) Aceito para publicação em 9 de fevereiro de 2001.

Extraído da Dissertação de Mestrado apresentada pelo primeiro autor à Universidade Federal de Viçosa (UFV), Viçosa, MG.

Trabalho financiado pela Fapemig e UFV.

(2) UFV, Dep. de Fitotecnia, CEP 36571-000 Viçosa, MG. E-mail rsantos@mail.ufv.br, fsilva@bol.com.br, vwcasali@mail.ufv.br (3) UFV, Dep. de Matemática. E-mail: dpi@ufv.br bos orgânicos tem aumentado nos últimos anos, graças principalmente aos elevados custos dos adubos minerais e aos efeitos benéficos da matéria orgânica em solos intensamente cultivados com métodos convencionais (Asano, 1984; Rodrigues, 1990).

As altas produtividades obtidas com o uso intensivo de capital, de fertilizantes inorgânicos e de agrotóxicos têm sido questionadas não só por suas contradições econômicas e ecológicas, mas também por desprezar aspectos qualitativos importantes da produção vegetal (Santos, 1993; Santos et al., 1994).

A alface é a hortaliça folhosa mais consumida no País, e apresenta grande resposta à adubação 
nitrogenada (Kiehl, 1985; Smith \& Hadley, 1989) e a altos teores de água no solo (Maciel, 1968), e possui grande potencial de produção com adubos orgânicos. Paralelamente, a adubação orgânica presta-se à reciclagem de resíduos rurais, o que possibilita maior autonomia dos produtores em face do comércio de insumos, e apresenta grande efeito residual (Marchesini et al., 1988; Smith \& Hadley, 1988, 1989; Vidigal et al., 1995a).

O solo ideal para o cultivo dessa hortaliça é o areno-argiloso, rico em matéria orgânica e com boa disponibilidade de nutrientes; para maior produtividade, é necessário o uso de insumos que melhorem as condições físicas, químicas e biológicas do solo (Vidigal et al., 1995a). A matéria orgânica adicionada ao solo na forma de adubos orgânicos, de acordo com o grau de decomposição dos resíduos, pode ter efeito imediato no solo, ou efeito residual, por meio de um processo mais lento de decomposição. Em trabalhos realizados com essa hortaliça foram observados aumentos na produção e nos teores de nutrientes nas plantas, após a aplicação de adubos orgânicos (Rodrigues, 1990).

O objetivo deste trabalho foi estudar o efeito residual da aplicação de composto orgânico, na presença e na ausência de adubo mineral, sobre o crescimento e produção da alface.

\section{Material e Métodos}

O experimento foi conduzido na Universidade Federal de Viçosa, em Viçosa, MG, em solo Podzólico VermelhoAmarelo, fase terraço. Os tratamentos foram arranjados em fatorial $5 \times 2$, no delineamento em blocos casualizados, com quatro repetições, e cinco doses de composto orgânico com as seguintes características: $17,38 \%$ de C orgânico; $1,92 \%$ de $\mathrm{N}$ total; $1,30 \%$ de $\mathrm{P} ; 1,58 \%$ de $\mathrm{K} ; 2,48 \%$ de $\mathrm{Ca}$ e $0,42 \%$ de $\mathrm{Mg}$, determinados por métodos descritos por Kiehl (1985). As doses foram 0,0, 22,8, 45,6, 68,4 e $91,2 \mathrm{t} / \mathrm{ha}$ de matéria seca, aplicadas no transplante das mudas, na presença e na ausência de adubo mineral O composto orgânico foi obtido a partir da mistura de capim-guatemala (Tripsacum fasciculatum) picado, bagaço de cana moído, palha de feijão, e cama de aviário. A adubação mineral seguiu recomendações da Comissão de Fertilidade do Solo do Estado de Minas Gerais (1989), e consistiu da aplicação de $643 \mathrm{~kg} / \mathrm{ha}$ da fórmula 4-14-8, aplicados na época do transplante, e duas adubações em cobertura aos 15 e 30 dias com $30 \mathrm{~kg} /$ ha de $\mathrm{N}$ (sulfato de amônio). A análise do solo indicou a seguinte composição: $\mathrm{pH}$ em água de 5,$87 ; 118,38 \mathrm{mg} / \mathrm{dm}^{3}$ do $\mathrm{P} ; 142,5 \mathrm{mg} / \mathrm{dm}^{3}$ de $\mathrm{K} ; 3,43 \mathrm{cmol}_{\mathrm{c}} / \mathrm{dm}^{3}$ de $\mathrm{Ca} ; 2,52 \mathrm{cmol}_{\mathrm{d}} / \mathrm{dm}^{3} \mathrm{de} \mathrm{H}+\mathrm{Al}$; $7,26 \mathrm{cmol}_{\mathrm{C}} / \mathrm{dm}^{3} \mathrm{de} \mathrm{CTC} ; 66,00 \% \mathrm{de} \mathrm{V} ; 0,00 \mathrm{cmol}_{\mathrm{C}} / \mathrm{dm}^{3} \mathrm{de}$ $\mathrm{Al} \mathrm{e} 16,2 \mathrm{~g} / \mathrm{kg}$ de M.O. Blocos adicionais com os mesmos tratamentos foram estabelecidos para determinar a curva de crescimento, expressa por meio do acúmulo de matéria fresca $\left(\mathrm{g} / \mathrm{m}^{2}\right)$, com vistas aos dias após o pegamento das mudas (DAP). Nesse último caso, as fertilizações constituíram a parcela, e os dias após o pegamento, as subparcelas.

Utilizou-se a cultivar Babá, no espaçamento de $0,25 \times 0,25 \mathrm{~m}$. As parcelas consistiram de $1,50 \times 1,50 \mathrm{~m}$ perfazendo um total de $2,25 \mathrm{~m}^{2}$; o primeiro cultivo foi conduzido de $28 / 8 / 90$ a $14 / 10 / 90$. Ao seu término, foram coletadas amostras de solo nas parcelas (quatro subamostras/parcela até $10 \mathrm{~cm}$ de profundidade), e procedeu-se à determinação dos teores de soma de bases $\left(\mathrm{cmol}_{\mathrm{d}} / \mathrm{dm}^{3}\right), \mathrm{P}\left(\mathrm{mg} / \mathrm{dm}^{3}\right)$ e CTC $\left(\mathrm{cmol}_{\mathrm{d}} / \mathrm{dm}^{3}\right)$, segundo o método descrito por Vettori (1969).

Para avaliação do efeito residual dos tratamentos aplicados, um segundo cultivo foi realizado 30 dias após a colheita do primeiro (14/11/90). Neste segundo ciclo de cultivo, foram avaliados o ganho de peso (g/planta) e a produtividade $\left(\mathrm{g} / \mathrm{m}^{2}\right)$ da matéria fresca e de matéria seca ( $\mathrm{kg} / \mathrm{ha})$, após secagem em estufa com ventilação de ar forçada por 48 horas, a $60^{\circ} \mathrm{C}$. Os dados foram coletados semanalmente, a partir do sétimo dia após o pegamento das mudas, até o 28 o dia, para determinação da curva de crescimento e da produção final na colheita, em quatro plantas/ parcela. Os dados foram submetidos à análise de variância, seguida de análise de regressão polinomial.

\section{Resultados e Discussão}

Houve efeito residual do composto orgânico, com resultados no crescimento e na produção semelhantes aos do primeiro cultivo (Santos et al., 1994). Os efeitos residuais das doses do composto orgânico somente verificaram-se após 14 DAP, ocorrendo maior acúmulo de matéria fresca com o incremento das doses (Figura 1).

A produção de matéria fresca cresceu linearmente com o incremento das doses de composto orgânico: $\mathrm{Y}\left(\mathrm{g} / \mathrm{m}^{2}\right)=1.704+14,72 \mathrm{ORG} ; \mathrm{R}^{2}=0,92$. Adose de $91,2 \mathrm{t} / \mathrm{ha}$ de MS propiciou 3.040,8 $\mathrm{g} / \mathrm{m}^{2}$ de matéria fresca. A produção de matéria seca também aumentou linearmente com as doses de composto orgânico: $\mathrm{Y}(\mathrm{kg} / \mathrm{ha})=3,2921+0,6508 \mathrm{ORG} ; \mathrm{R}^{2}=0,70$. Efeitos lineares crescentes de doses de adubos orgânicos sobre a produção de matéria fresca, da matéria seca e 
do diâmetro da planta em cultivos sucessivos da alface também foram observados por Vidigal et al. (1995a, 1995b).

Conforme relatado por Santos et al. (1994), doses crescentes de composto orgânico elevaram a produtividade da cultura. As maiores produções obtidas com as doses crescentes de composto orgânico podem ser atribuídas à melhoria das características químicas e físico-químicas do solo. Ao final do primeiro cultivo, a mineralização do material orgânico aumentou os teores de bases trocável, o teor de fósforo e a capacidade de troca catiônica do solo (Figura 2).

O aumento na CTC certamente contribuiu para atingir os resultados observados, à semelhança dos relatos de Meek et al. (1982). Paralelamente ao aumento nos teores de bases trocáveis, de P e da CTC, a contínua liberação de $\mathrm{N}$ pela mineralização do material orgânico ajusta-se melhor às necessidades da alface do que o fornecimento de formulações solúveis prontamente disponíveis, conforme relatado por Smith \& Hadley (1988).

Outro efeito da aplicação do composto orgânico foi atribuído ao suprimento de macro e micronutrientes, de forma equilibrada, uma vez que maiores produtividades de diversas hortaliças foram obtidas com doses intermediárias de nutrientes do que com maiores quantidades de macronutrientes isoladamente (Silva Júnior, 1987; Marchesini et al., 1988).

Não houve efeito da adubação mineral na produtividade do primeiro cultivo (Santos et al.,1994), nem do segundo cultivo, não se constatando, portanto, efeito residual deste fertilizante.

O solo adubado organicamente repôs, ao menos parcialmente, os nutrientes exportados ao final do

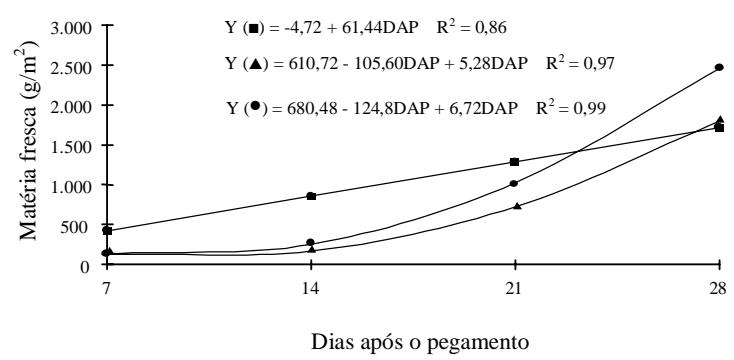

Figura 1. Efeito de dias após o pegamento (DAP) sobre o acúmulo de matéria fresca na parte aérea de plantas de alface, em três doses de composto orgânico (0 (- $)$, 45,6 (ム) e 91,2 t/ha $(\bullet)$ ), na ausência de adubo mineral. primeiro cultivo, como pode ser constatado pelos altos teores de $\mathrm{P}$ disponível, pelas bases, e pela alta CTC, presentes após a primeira colheita (Figura 2) e que propiciaram efeitos residuais progressivos com o aumento das doses aplicadas, verificados de 80 a 110 dias após incorporação do composto, à se-
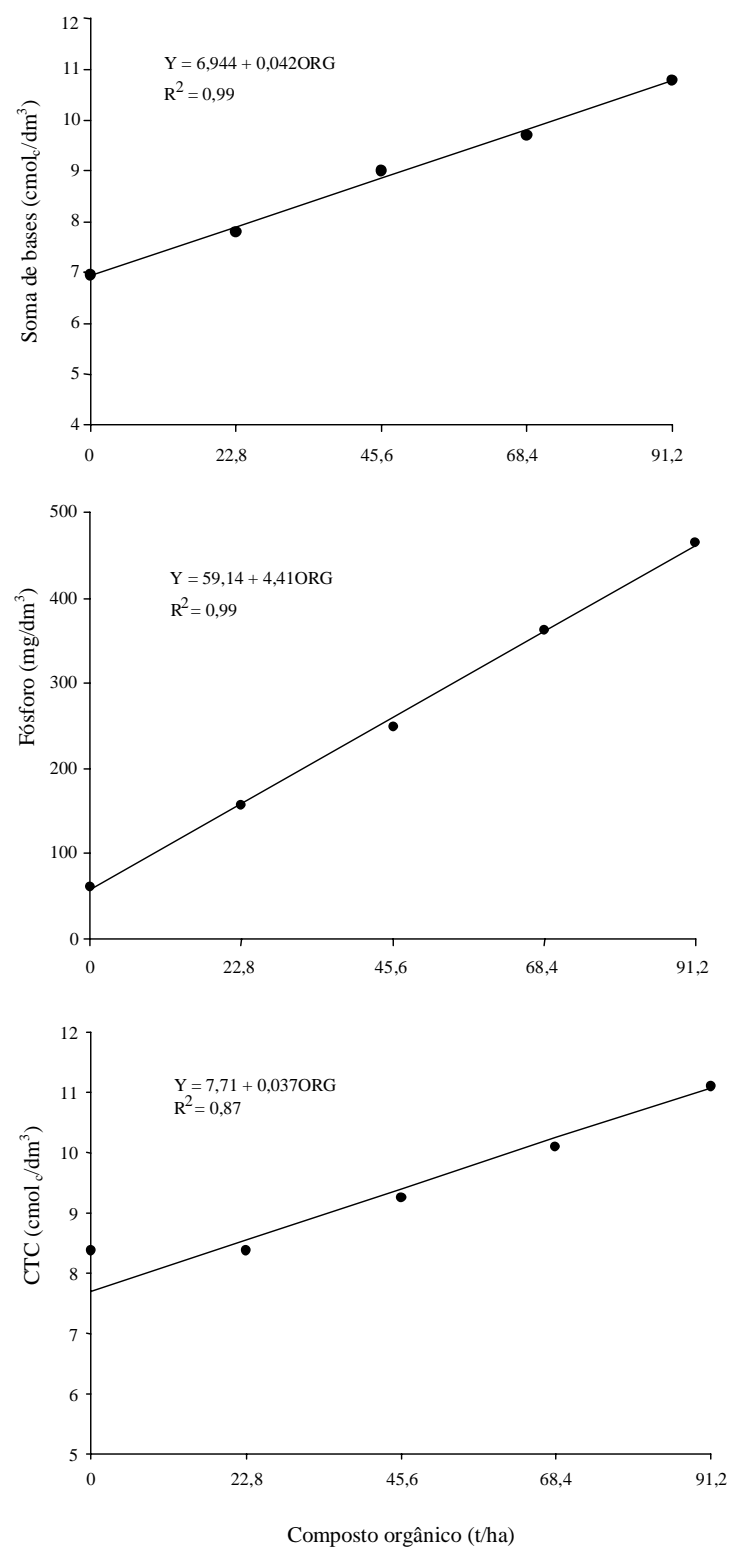

Figura 2. Efeito das doses de composto orgânico (ORG) na soma de bases, no teor de fósforo e na capacidade de troca catiônica do solo, ao final do primeiro cultivo. 
melhança dos resultados relatados por Peavy \& Greig (1972), Schuphan (1974), Asano (1984) e Marchesini et al. (1988). Vidigal et al. (1995a, 1995b) observaram que efeitos residuais de composto orgânico têm sido verificados em até três cultivos sucessivos, em que os teores de macronutrientes atingiram níveis adequados no tecido foliar.

Neste sentido, Smith \& Hadley (1989) relatam que parte do $\mathrm{N}$ presente em adubos orgânicos resiste à rápida mineralização, e torna-se disponível somente às culturas subseqüentes; e Marchesini et al. (1988) relatam, ainda, que os incrementos de produtividade proporcionados por adubos orgânicos, embora menos imediatos e marcantes do que os obtidos com adubos minerais, apresentam maior duração, provavelmente pela liberação mais progressiva de nutrientes e pelo estímulo do crescimento radicular; os mesmos autores concluíram, ainda, que o uso de composto não só supre as plantas com quantidades consideráveis de nutrientes, mas contribui para manter a fertilidade natural, o que envolve os ciclos biológicos dos nutrientes nas terras cultivadas, prevenindo sua exaustão.

\section{Conclusões}

1. A adubação com composto orgânico propicia efeito residual sobre produção de alface, cultivada de 80 a 110 dias após a aplicação do composto.

2. O aumento de adubo orgânico aumenta os teores de bases e de P, e a CTC do solo.

3. O adubo mineral não propicia efeito residual sobre a produção de alface.

\section{Referências}

ASANO, J. Effect of organic manures on quality of vegetables. Japan Agricultural Research Quarterly, Ibaraki, v. 18, n. 1, p. 31-36, 1984

COMISSÃO DE FERTILIDADE DO SOLO DO ESTADO DE MINAS GERAIS (Lavras, MG). Recomendação do uso de corretivos e fertilizantes no Estado de Minas Gerais: $4^{a}$ aproximação. Lavras, 1989. 176 p

KIEHL, E. J. Fertilizantes orgânicos. Piracicaba: Agronômica Ceres, 1985. 492 p

MACIEL, R. F. P. Estudo sobre a influência do espaçamento, níveis de adubação, irrigação e adubação na cultura da alface (Lactuca sativa $\mathbf{L}$.). Viçosa, MG Universidade Rural do Estado de Minas Gerais, 1968. 48 p Dissertação de Mestrado.
MARCHESINI, A.; ALLIEVI, L.; COMOTTI, E.; FERRARI, A. Long-term effects of quality-compost treatment on soil. Plant and Soil, Dordrecht, v. 106, p. 253-261, 1988.

MEEK, B.; GRAHAM, L.; DONOVAN, T. Long-term effects of manure on soil nitrogen, phosphorus, potassium, sodium, organic matter and water infiltration rate. Soil Science Society of America Journal, Madison, v. 46, p. 1014-1019, 1982.

PEAVY, W. S.; GREIG, J. K. Organic and mineral fertilizers compared by yield, quality and composition of spinach. Journal of the American Society for Horticultural Science, Alexandria, v. 97, n. 6, p. 718-723, 1972

RODRIGUES, E. T. Efeitos das adubações orgânica e mineral sobre o acúmulo de nutrientes e sobre o crescimento da alface (Lactuca sativa L.). Viçosa, MG: UFV, 1990. 60 p. Dissertação de Mestrado.

SANTOS, R. H. S. Crescimento, produção e qualidade de alface (Lactuva sativa) cultivada com composto orgânico. Viçosa, MG: UFV, 1993. 114 p. Dissertação de Mestrado.

SANTOS, R. H. S.; CASALI, V. W. D.; CONDÉ, A. R.; MIRANDA, L. C. G. de. Qualidade de alface cultivada com composto orgânico. Horticultura Brasileira, Brasília, v. 12, n. 1, p. 29-32, 1994.

SCHUPHAN, W. Nutritional value of crops as influenced by organic and inorganic fertilizer treatments. Qualitas Planttarum, Dordrecht, v. 23, n. 4, p. 333-358, 1974.

SILVA JÚNIOR,A. A. Adubação mineral e orgânica em repolho: II. Concentração de nutrientes na folha e precocidade. Horticultura Brasileira, Brasília, v. 5, n. 1, p. 15-17, 1987.

SMITH, S. R.; HADLEY, P. A comparison of organic and inorganic nitrogen fertilizers: their nitrate-N and ammonium-N release characteristics and effects on the growth response of lettuce (Lactuca sativa $\mathrm{L}$. cv. Fortune). Plant and Soil, v. 115, n. 1, p. 135-144, 1989.

SMITH, S. R.; HADLEY, P. A comparison of the effects of organic and inorganic nitrogen fertilizers on the growth response of summer cabbage (Brassica oleracea var capitata $\mathrm{cv}$. Hispe F1). Journal of Horticultural Science, Ashford, v. 63, n. 4, p. 615-620, 1988

VETTORI, I. Métodos de análise de solo. Rio de Janeiro: Ministério da Agricultura, 1969. 24 p.

VIDIGAL, S. M.; RIBEIRO, A. C.; CASALI, V. W. D.; FONTES, L. E. F. Resposta da alface (Lactuca sativa L.) ao efeito residual da adubação orgânica: I. Ensaio de campo. Revista Ceres, Viçosa, v. 42, n. 239, p. 80-88, 1995a. VIDIGAL, S. M.; RIBEIRO, A. C.; CASALI, V. W. D.; FONTES, L. E. F. Resposta da alface (Lactuca sativa L.) ao efeito residual da adubação orgânica: II. Ensaio em casa de vegetação. Revista Ceres, Viçosa, v. 42, n. 239, p. 89-97, 1995 b. 